\title{
Analysis of pricing mechanisms in a microgrid with active electric energy storages
}

\author{
Vsevolod Korepanov ${ }^{1, *}$ and Tatiana Vaskovskaya ${ }^{1,2}$ \\ ${ }^{1}$ V.A.Trapeznikov Institute of control sciences of RAS, 117997, Profsoyuznya 65, Moscow, Russia \\ ${ }^{2}$ National Research University "Moscow Power Engineering Institute" 111250, Krasnokazarmennaya 14, Moscow, Russia
}

\begin{abstract}
A model of power market with a simple market clearing price mechanism on microgrid is considered. Market participants are consumers, prosumers with energy storage systems (ESS), and the power company as a guaranteed supplier. Prosumers charge their ESS in the first off-peak period with low prices from the power company and can decrease their demand or sell energy in the market through discharging the ESS in the second peak period. The strategic behavior of participants and their influences on a system state are postulated as the goal of work. Some use cases and model elements are discussed.
\end{abstract}

\section{Introduction}

The microgrid idea allows us to think about creating a micro market - local electricity market at the lowest level, the household level. It arose in connection with the growing popularity and falling prices for renewable sources (solar, wind, etc.) and energy storage (chemical batteries, hydroelectric storage, etc.) - let us call all these elements "microenergetics". At the same time, the critical factor from which the desire to create a market arises is that a household can become an electricity producer rather than just a consumer, i.e., it can transfer the excess of its sources of energy to other households in the microgrid. Such a network participant is usually called a prosumer.

The interest of large energy companies to research and create microgrid technologies and subsidizing purchases by households is related to the smoothing of temporary peaks in energy consumption connected with microenergetics. At the same time, when using only energy storage systems (ESS), the total consumption of the prosumer increases due to the inevitable loss of power in work processes of the ESS. For the energy company, there is a double interest: smoothing peaks + growth in total consumption of customers who use only energy storage devices.

In [1], a hierarchy of models for analysing price mechanisms in energy markets was proposed from a strategic point of view - decomposition of complex control problem into a number of elementary, basic problems [2]. There are cases with fixed demand and active producers, with active demand, with active parties owning several producers and/or consumers, and various mechanisms of pricing and competition. Two-node models with electricity transfer between nodes are considered separately. The models are analysed using game theory, mechanism design, and business games with dedicated students.

In this paper, we aim at a similar analysis with an emphasis on other aspects: microgrid and prosumer. We will try to outline the simplest model that will contain the required properties and based on the models in [3] and [4]. Basically our prosumers modelling similar to [4] and [5] but our goal and market modelling are slightly different. In [4], the market price is a linear function and a future price is a random variable, the power company choose a base market price as a leader in Stackelberg game. In [5] the market price is based on historical data, the ESS and a peer-to-peer market of prosumers are compared to basic interaction of prosumers with the power grid.

\section{Model}

Let us consider a consumers set $C=\{1, \ldots, m+n\}$, a prosumers set $P=\{1, \ldots, n\} \subset C$ and one «big» guaranteed supplier $M$. Let us consider the local market model with the standard market mechanism for determining the Market Clearing Price (MCP) [6]. The supplier $M$ can provide electricity for all needs of the local electricity market.

Further, firstly, due to the presence of prosumers, both consuming and producing processes should be considered. We should not fix the overall demand. Secondly, since the prosumer separate consumption and production of energy in time, it is necessary to consider at least two periods of time, for example, the off-peak and the peak periods or, conventionally, the first and second periods $t \in\{1,2\}$. Note that the temporal aspect was not considered earlier in [2]. The peak period is characterized by an increase in consumer energy demand

\footnotetext{
Corresponding author: moskvo@ya.ru
} 
and price and an increase in the price of the single supplier $M$. According to [7], the use of the ESS in the wholesale market makes it possible to flat locational marginal prices. However, a microenergetics ESS can produce such an effect only with massive deployment. In our work, the prices of the single supplier remain different.

Each consumer (including a prosumer) $i \in C$ has a demand profile in each period: $d_{i}(1)<d_{i}(2)$ and the maximum prices at which he is ready to buy energy: $p_{i}(1) \leq p_{i}(2)$. A prosumer can either reduce its demand during the period by discharging the ESS and increase it by charging the ESS. The prosumer $i$ has ESS with a capacity $S_{i}$. Then the prosumer can change its power demand from $\left(d_{i}(t)-S_{i}\right)$ to $\left(d_{i}(t)+S_{i}\right)$. The prosumer can also produce some electricity volume $g_{i}(t) \in\left[0, S_{i}\right]$ by price $p_{i}^{g}(\mathrm{t})$ as a generator in the market.

The goal function of the prosumer is the total of electricity sales revenue, the cost of purchasing electricity in the local market, and the fixed cost of maintaining microenergetic devices, while the devices can help both increase revenue and reduce costs:

$$
f_{i}\left(s_{i}, g_{i}\right)=\sum_{t=1}^{2}-\left(d_{i}(t)+s_{i}(t)-g_{i}(t)\right) P(t)-2 c S_{i},
$$

where $P(t)$ is the price of energy in the micro market during the period $t, s_{i}(t) \in\left[-S_{i}, S_{i}\right]$ is the volume of energy discharged from the ESS (if the value is negative) or the volume of energy charged from the power grid (if the value is positive) in the period $t, g_{i}(t)$ is energy of the ESS passed to the market as a generator, $c$ is the unit cost of maintaining the ESS of volume $S_{i}$ for one period.

Denote the initial stored energy as $s_{i}(0) \in\left[0, S_{i}\right]$. Then the following physical restrictions can be introduced:

$$
\begin{gathered}
0 \leq s_{i}(0)+s_{i}(1)-g_{i}(1) \leq S_{i} \\
0 \leq s_{i}(0)+s_{i}(1)-g_{i}(1)+s_{i}(2)-g_{i}(2) \leq S_{i}
\end{gathered}
$$

Note, that charging and discharging ESS in the same period does not make sense. Suppose a consumer wants to get profit from the charge and the subsequent discharge from the ESS. Since energy buying/selling in this period will occur at the same price, the player will not gain anything from this operation in total. Moreover, taking unavoidable energy losses in ESS into account makes it completely unprofitable. Therefore, we don't consider the case $g_{i}(t) s_{i}(t)>0$.

The goal function of the energy company should, in addition to accounting for an income from sales of energy on microgrids, consider the reduction in consumption during the peak period, since even a small peak-consumption reduction in each microgrid will have a significant cumulative effect, which is not captured by the energy company goal function from [4], for example.

\section{Examples}

Let us consider the example in figure 1.

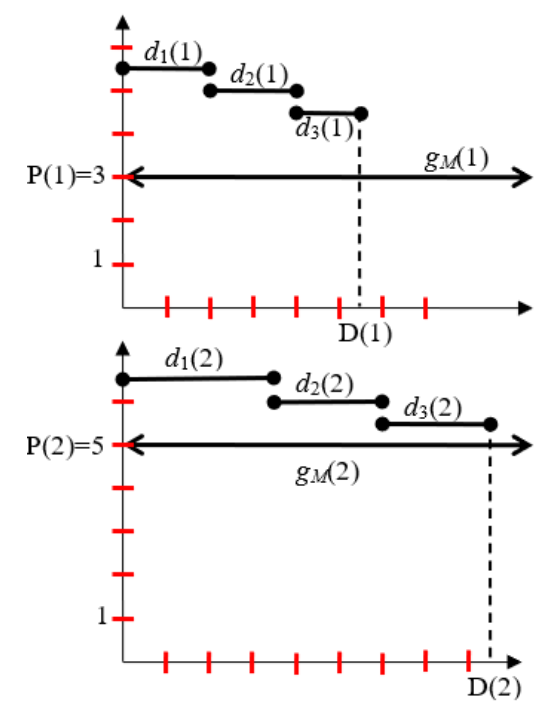

Fig. 1. Example with 3 consumers

In general, since consumption and prices are higher in the second period, then the market price in the second period is higher. The values of the goal functions of consumers 1 and 2 are equal to -23.5 and -18.5 respectively (without considering fixed costs for the ESS).

Suppose that consumers 1 and 2 bought the ESS and became prosumers. They charge them in period 1 because of the low price of the supplier $M$, and in period 2 they use it to reduce their demand or to sell a part of the ESS energy in the market as a generator. It seems reasonable that it is profitable for a prosumer to first fully meet his demand in period 2 and to give an ESS surplus to the market. Consider an example $d_{1}(2)=3.5$, $S_{1}=5.5, d_{2}(2)=2.5, S_{2}=3.5, d_{3}(2)=2.5$. Suppose that prosumers 1 and 2 fully cover their demand in period 2 , and they sell the excess as producers: $g_{1}(2)=2$ and $g_{2}(2)$ $=1$ in the market at a price of 3.5. Then the market price in the second period will be $P(2)=3.5$. This situation is shown in figure $2 \mathrm{a}$.

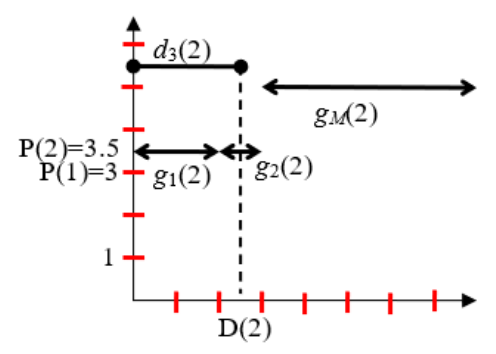

(a)

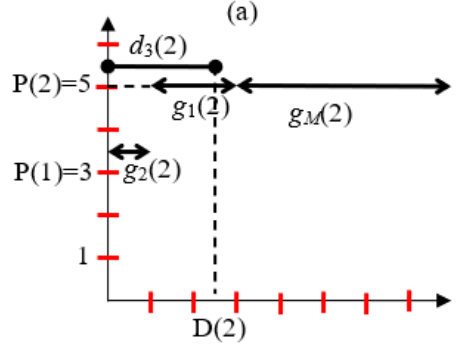

(b)

Fig. 2. Situation change due to ESS in period 2

In this situation, the goal functions of the prosumers are -15.5 and -14.75 taking into account the increased 
consumption in period 1 for charging the ESS (but excluding fixed costs). From a game-theoretic point of view, this situation is unstable: prosumers can increase the value of the goal functions by raising the market price up to 5 by raising the price for their generation $g_{i}$. In this case, it is more profitable for prosumer 2 to set a price lower than prosumer 1 - then he will be able to sell the entire volume $g_{2}(2)$. For example, the Nash equilibrium (one of) will be the situation shown in figure $2 \mathrm{~b}$. In this situation, prosumer 2 sells the entire available volume of energy, while prosumer 1 does not. In order to sell it, the price for $g_{1}(2)$ must be lower than 3.5, which will not bring him an increase in the goal function.

At the same time, we see that the market price has not become lower, but the goal functions of prosumers have improved, and overall demand has decreased, which is good for the energy company and the social welfare increases. Moreover, in the second peak period, this market does not need additional external energy at all since the total generation of the ESS exceeds the total demand.

Let us consider situation when a prosumer can increase the market price through decreasing its capacity - so called "power withdrawal". Prosumer 2 in figure $3 \mathrm{c}$ decreases its capacity from $2 \mathrm{~kW}$ to $1 \mathrm{~kW}$ in figure $3 \mathrm{~d}$. As a result, the market price increased from 3.5 to 5 .

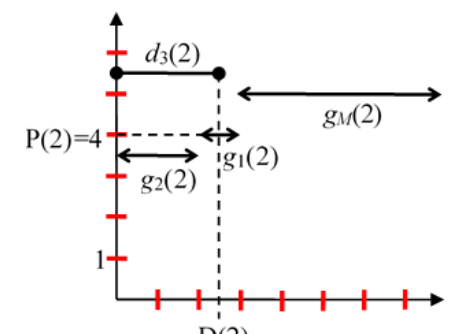

(c)

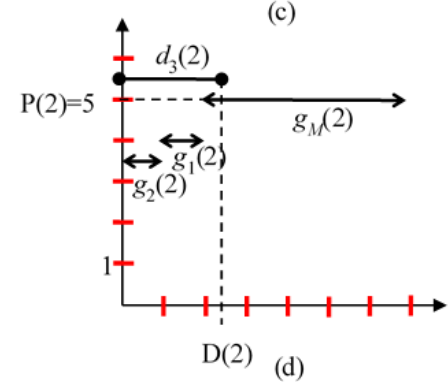

Fig. 3. Start situation (c) and power withdrawal (d)

\section{Conclusions}

We are constructing a basic model of a microgrid market to investigate game-theoretic behaviour of prosumers. There are many possibilities to expand the model for real-life cases:

- to consider more periods, prosumers,

- to take into account ESS physic: e.g., $3 *$ charging speed $\approx$ discharging speed,

- to choose a subsidy model and other pricing mechanisms see [6, Section 3.3].

In each case, we should describe prosumers' behaviour and equilibrium; therefore, understanding of the utility and goal function of active participants is crucial.

For example, an increase in consumption cannot reduce the price in the market, and an increase in generation cannot increase the price. So, when we consider the model with several prosumers, only because of their number, a situation may arise when prices in the off-peak and peak periods converge so much that the use of storage would be economically impractical. Then perhaps a game-theoretic situation such as a game of entering the market [8] will arise, where prosumers will have to coordinate.

We are grateful to the project team of https://energy.ipu.ru for their support.

This work is partially supported by the Russian Scientific Foundation, project no. 16-19-10609.

\section{References}

1. V. Chirkin, M. Goldstein, A. Gorbunov, M. Goubko, V. Korepanov, N. Korgin, ... \& T. Vaskovskaya et al., IFAC-PapersOnLine 49.32, 1318 (2016)

2. V.N. Burkov et al. Mechanism Design and Management: Mathematical Methods for Smart Organizations:(for Managers, Academics and Students) (Nova Publishers, 2013)

3. Z. Hu Energy Storage for Power System Planning and Operation. (John Wiley \& Sons, 2020)

4. G. El Rahi, S.R. Etesami, W. Saad, N. Mandayam, H. Vincent Poor, IEEE Transactions on Smart Grid 10.1, 702-713 (2017)

5. J.M. Zepter, A. Lüth, P.C. del Granado, R. Egging, Energy and Buildings 184, 163-176 (2019)

6. A. Sumper (ed.). Micro and Local Power Markets. (John Wiley \& Sons, Incorporated, 2019)

7. T.A. Vaskovskaya, Energy System Research 2.2, 2840 (2019)

8. R. Selten and W. Guth. Equilibrium Point Selection in a Class of Market Entry Games (In Games, Economic Dynamics, and Time Series Analysis; Diestler, M., Furst, E., Schwadiauer, G., Eds.; Physica-Verlag: Wien-Wurzburg, Austria-Germany, 1982) 